\title{
FECUNDIDAD, ¿DECISIÓN ABSOLUTA DE LA MUJER ACTIVA?
}

FECUNDITY, ABSOLUTE DECISION OF THE ACTIVE WOMAN?

1 Universidad Católica de Santiago de Guayaquil. eduardo.guzman01@cu.ucsg.edu.ec

2 Universidad Católica de Santiago de Guayaquil. maria.salazar02@cu.ucsg.edu.ec

En esta investigación se estudió la relación que existe entre la inserción de la mujer en el mercado como ente activo (ya sea obteniendo una profesión mediante la realización de estudios universitarios, o desarrollando una actividad laboral dependiente 0 independiente) y la decisión de tener menos hijos. Esta relación supuesta hasta el momento, es especialmente notable en los países más desarrollados, en donde la inclusión de la mujer al mercado empezó mucho tiempo atrás. Esta investigación se realizó con datos obtenidos del Banco Mundial con un rango de 19 años. El trabajo se desarrolló a través de la epistemología popperiana y se basó en la comprobación de la hipótesis plasmada a partir de la pregunta de investigación.

PALABRAS CLAVE: inserción laboral, inclusión laboral, tasa de natalidad, fecundidad.

INTRODUCCIÓN Uno de los principales cambios dentro de la población económicamente activa de las naciones, ha sido justamente la inserción paulatina y en aumento, de las mujeres al mercado laboral. Una de las razones para su inserción ha sido sin duda la necesidad de colaborar con el sustento familiar, pues los ingresos individuales no satisfacen las necesidades familiares. La razón anterior se sustenta en el resultado positivo del planteamiento hipotético del estudio realizado por Easterlin (1968), en el que
ABSTRACT In this research, the relationship between the inclusion of women in the market as active is being studied (either by obtaining a profession through university studies, or developing a dependent or independent labor activity) and the decision to have fewer children. This supposed reality so far is particularly noticeable in the more developed countries, where the inclusion of women into the market began long ago. This research was performed with data obtained from the World Bank within a range of 19 years. The work is developed through Popperian epistemology and is based on checking the hypothesis captured from the research question.

KEYWORDS: job placement, job inclusion, birth rate, fertility.

relaciona la tasa de fertilidad con el índice de ingreso relativo intergeneracional, "calculado a partir de la proporción entre el ingreso actual del esposo y el nivel de ingreso experimentado por éste durante su adolescencia en su hogar de crianza”(Alvis-Guzmán, Yánez-Contreras, Quejada-Pérez, Acevedo-González, y Del RíoCarrasquilla, 2010, p.93).

La educación ocupa también un lugar significativo entre los motivos para la inserción laboral femenina a través de los años. Estudios como los realizados por Álvarez (2002) y Bratti 
(2003), muestran que la educación es un factor clave para la decisión de tener o no hijos. Álvarez (2002) encontró que la participación de la mujer en el mercado laboral en España, "reduce la probabilidad en un $68 \%$ de tener el primer hijo, un $80 \%$ de tener el segundo hijo, y un $78 \%$ para el tercero" (p. 215). Por su parte Bratti (2003), considera que la educación actúa a través de diferentes vías en relación a la fecundidad. Por ejemplo se encuentra la edad del matrimonio, afectación sobre el número de hijos que se tienen luego del matrimonio. El mayor nivel educativo incrementa el enganche de las mujeres en el mercado de trabajo (Álvarez, 2002), y las mujeres más educadas posponen tener hijos (Rindfuss, Morgan, \& Offutt, 1996).

PREGUNTA DE INVESTIGACIÓN ¿Cómo incide la activación de la mujer en el mercado laboral y universitario en la variación de la tasa de natalidad?

REVISIÓN DE LA LITERATURA

Esta interrogante ha sido abordada en diferentes estudios, espacios y tiempos, empezando con una inferencia de relación directa entre la inserción laboral de la mujer y la reducción de la tasa de natalidad. En este sentido, se revisarán algunos de los principales estudios sobre la problemática a nivel internacional.

En la revisión de la literatura correspondiente al tema destaca entre muchas, la investigación de Becker, quién además fue el autor de la “Teoría económica de la Fecundidad” utilizada en el presente estudio para probar la relación entre las variables indicadas en la pregunta de investigación. Becker en su libro "Tratado sobre la familia” (1981), presenta exploración en temas que estaban en aquella época, muy alejados del interés de la ciencia económica neoclásica. Entre ellos destacan, el matrimonio, la planificación familiar, entre otros. "Hace un especial énfasis en la economía de la familia y plantea a la reproducción en un esquema de asignación de recursos basado en la maximización de la utilidad dadas las restricciones de tiempo y renta (Picchio, 1994, p. 476)" (Anzorena, 2009,p.2).

Para Becker un hogar eficiente es aquel donde cada miembro se va a especializar en el hogar o en el mercado, según tenga mayores ventajas comparativas. Un punto importante que también expone en el tratado sobre la familia, es referente al cálculo económico de la cantidad de hijos a tener. Los hijos se entienden como bienes de consumo. Llega a la conclusión de que la demanda de hijos depende del precio relativo de los hijos y de la renta plena.

Esto determina finalmente el cambio entre cantidad de hijos y la calidad de vida que se desea que estos tengan, haciendo énfasis que esto aplica a padres con altos niveles de estudios, insertos en el mercado laboral, que proyectan tener un número de hijos acorde a la dedicación en tiempo, la renta, etc.

Un punto a destacar que también es abordado en parte por Becker y Barro, trata sobre el altruismo de los padres hacia los hijos, y resalta que este altruismo hace que se elija mesuradamente el número de hijos a tener, pensando en el consumo promedio y en la maximización de la utilidad dinástica. Dicha utilidad dinástica tiene sentido en función del traspaso generacional, descendencia (Becker \& Barro, 1986).

En un ejemplo básico, Becker (1960) intenta clarificar el altruismo y la calidad sobre la cantidad de hijos a tener en una familia. "Yo llamaré niños más caros de mantener "niños con alta calidad', igual como un Cadillac es llamado un auto de mayor calidad que un Chevrolet" (p. 211).

Los supuestos básicos de la Teoría Económica de la Fertilidad son: 1) Los hijos son bienes de consumo duradero; 2) Altruismo de los padres hacia los hijos; 3) Maximización de la utilidad dinástica y 4) La fertilidad es determinada por la interacción entre calidad y cantidad de hijos en función de la utilidad (Becker, \& Barro, 1986) (Tijerina, 1999).

Otras consideraciones relevantes de la Teoría Económica de la Fertilidad son analizadas y sintetizadas por (Bonilla, García, \& Mendoza, 2009) y detalladas a continuación:

a. El mejoramiento en el capital humano de la mujer incrementa el costo del tiempo, por ende el precio del servicio de crianza ofrecido al niño. El deseo de tener un niño se reduce.

b. Además, la revalorización del tiempo de la madre la incentivará a participar activamente en el mercado laboral.

c. Un mayor nivel de educación le permite a la mujer aplazar su casamiento. Por lo que mujeres, con elevado nivel educacional no utilizan todo su período de fertilidad. Por consiguiente, tiene menos hijos en comparación con mujeres menos educadas. Asimismo, la educación reduce los potenciales compañeros y aumenta el tiempo requerido para escoger el individuo más adecuado. Por lo que la edad de matrimonio y el nivel 
de educación pueden determinar la tendencia de la fertilidad.

d. Otro importante determinante de la fertilidad familiar es el conocimiento anticonceptivo. Acceso a información y uso de métodos anticonceptivos como de servicios médicos, pueden ser críticos a la hora de disminuir el crecimiento poblacional en países de ingresos-bajos. (p. 3)

Schultz (1973) considera que, uno de los avances más relevantes en el análisis económico fue el tratamiento del tiempo humano como un concepto más amplio (Becker, 1965), que no se limita únicamente al trabajo en el mercado laboral, sino que también es aplicable al trabajo en el hogar. Cuidar y criar un niño son tareas tiempo intensivas realizadas por la madre. El autor sostiene que la crianza de niños difiere en términos de insumo de tiempo y de bienes de acuerdo con la edad de los niños. Un niño muy pequeño es altamente trabajo intensivo en términos del tiempo de la madre, a medida que crece, se vuelve menos tiempo intensivo y más costoso en términos de otros recursos familiares necesarios para su educación y otras actividades que va desarrollando. El costo depende de la edad de los hijos. (Cáceres, Elías y Fernández, 2005, p.3).

En el artículo de Mesa y Junca (2011) se expone la teoría de la transición demográfica, abordada por Thompson (1929), Landry (1934) y Notestein (1953), quienes clasificaron la evolución de la población en tres etapas de acuerdo con su comportamiento:

1. Fase 1.- Etapa de alta tasa de natalidad y alta tasa de mortalidad, por lo que se presenta un bajo crecimiento poblacional.

2. Fase 2.- Se mantiene la alta natalidad, mientras que la mortalidad comienza a descender. El crecimiento poblacional es alto y esta etapa se conoce como de explosión demográfica.

3. Fase 3.- La tasa de natalidad desciende y coincide con una tasa de mortalidad baja y con poco cambio. El crecimiento poblacional es bajo. (p. 128-129).

En la actualidad el mundo está inmerso en la tercera fase, donde las tasas de natalidad y mortalidad descienden, teniendo una población cada vez más vieja. El análisis anterior se sustenta en que los padres disponen de una función de utilidad basada en los hijos y "en términos económicos esta función se logra maximizar justo cuando la utilidad marginal del último hijo sea igual a la desutilidad, o en otros términos, la satisfacción obtenida por él es negativa (Overbeek, 1974)" (Mesa y Junca, 2011, p. 130). "La utilidad, sin embargo, hace referencia a los beneficios esperados por los hijos, los cuales pueden ser: ver a los hijos como una fuente de placer, como fuente del ingreso familiar futuro y como un seguro de ancianidad de los padres" (Mesa y Junca, 2011, p. 130).

Leibenstein (1974), establece un modelo explicativo que relaciona los cambios producidos en la fecundidad con el desarrollo económico, diferenciando además los beneficios y los costes que producen los hijos:

BENEFICIOS

1. Consumo entendido como satisfacción personal.

2. Trabajo o renta que el niño, cuando entre en la fuerza laboral de trabajo, aportará a la familia.

3. Seguridad referida al apoyo que el hijo prestará a sus padres cuando éstos estén viejos.

COSTES

1. Coste directo de educación, ropa, comida, etcétera.

2. Coste indirecto. Pérdida de oportunidades debido al tiempo dedicado al cuidado y educación del niño (Aguinaga, 1983, p. 87).

En un estudio en el que se abordan temáticas relacionadas a la actividad laboral femenina y la decisión de ser madre o no, Marín (2002), realiza las siguientes reflexiones:

- El precio de mercado de la mujer es el elemento que en mayor medida va a determinar si la mujer decide ser activa o no; siendo a su vez el nivel educativo de la mujer, el elemento que determina el valor del salario sombra ${ }^{1}$.

- La probabilidad de que una mujer concreta pertenezca a la población activa, está relacionado de manera fuerte y positiva con su nivel educativo, sobre todo para las no solteras.

1 Precio estimado del tiempo de mercado de la mujer. 
- Para las mujeres no solteras el salario de su pareja y el número de hijos habidos son dos elementos que también se combinan a la hora de determinar su tasa de actividad, de tal forma que la probabilidad de que una mujer no soltera permanezca en la población activa está inversamente relacionada con el salario de su pareja o cónyuge.

- La participación laboral de la mujer no soltera está negativamente relacionada con el número de hijos, ya que la existencia de hijos en el hogar hace necesario un cierto tiempo para encargarse de su cuidado, alimentación etcétera, de tal modo que será necesario un salario superior para inducir a la mujer a abandonar el hogar e incorporarse al mercado laboral.

- Esto explica que sólo las mujeres con altos niveles de estudios, o aquellas otras para las que el salario de su marido es muy bajo, compatibilicen actividad laboral y maternidad. (p. 24).

La data a utilizar para probar la existencia de relación entre las variables a estudiar se obtuvo de las fuentes del Banco Mundial. Las variables más cercanas al estudio y que están disponibles en la base de datos son:

- Fuerza laboral femenina con educación terciaria (como \% de la fuerza laboral femenina).

- Tasa de natalidad, nacidos vivos en un año (por cada 1.000 personas).

Estas dos variables sitúan adecuadamente la relación que se quiere demostrar en esta investigación. Por un lado, la fuerza laboral femenina con educación terciaria encierra justamente la inserción de la mujer en el mercado con el nivel de educación obtenido, que le da mayores posiblidades de conseguir un empleo acorde a lo requerido.

Se han tomado los datos de ambas variables en el espacio de los países de la Unión Europea, entre los años 1993 al 2012. Se cuenta con los datos de 19 años. De acuerdo con Figueroa (2012), la epistemología popperiana sugiere que el conocimiento científico solo puede darse usando la lógica hipotética deductiva, solo construyendo teorías que intenten explicar el mundo real, y que sus implicaciones sobre las observaciones del mundo real se deriven de la lógica deductiva. Estas implicaciones son observables y constituyen hipótesis del comportamiento del mundo real y están destinadas a probarse. Si la teoría y la realidad coinciden, no se tienen razones para descartar la teoría, pero si no se cumple, la teoría debe falsearse.

Como existe la Teoría y además la data, se selecciona la celda 1 , utilizando la epistemología popperiana para probar la relación entre variables del estudio, mostrado en la Tabla 1.

\begin{tabular}{|c|c|c|}
\hline TEORÍA/DATA & DISPONIBLE & NO DISPONIBLE \\
\hline Disponible & $\begin{array}{c}\mathrm{x} \\
\text { Epistemología popperiana }\end{array}$ & \\
\hline № Disponible & & \\
\hline
\end{tabular}

Fuente: Tomado de Figueroa (2012).

MÉTODO ALPHA BETA

Se parte del conocimiento de la teoría y la data, luego de ello se debe representar gráficamente las relaciones existentes entre las variables del estudio, con la finalidad de reducir la realidad compleja a una abstracción que permita estudiarla y someter a falsación las proposiones planteadas.

PROPOSICIONES $\alpha$ : Teoría Económica de la Fecundidad $\alpha$ : La Teoría Económica de la Fecundidad aplicada en los países de la Unión Europea, en el período 1993-2012.

$\beta$ ': La tasa de natalidad disminuye a medida que se incrementa la fuerza laboral de la mujer con educación terciaria.

La matriz de causalidad se muestra a continuación en la Tabla 2.

TABLA 2. MATRIZ DE CAUSALIDAD VARIABLE ENDÓGENA VARIABLE EXó́GENA

\begin{tabular}{ll}
\hline Tasa de natalidad & fuerza laboral con educacion terciaria, mujeres \\
\hline
\end{tabular}

Fuente: Tomado de Figueroa (2012).

A partir de la metodología alfa beta, se plantea el diagrama representativo del proceso de análisis, como lo muestra la Figura 1 presentada por Figueroa (2012), en la cual se interrelacionan la variable exógena y endógena por medio del mecanismo mostrado a continuación. 


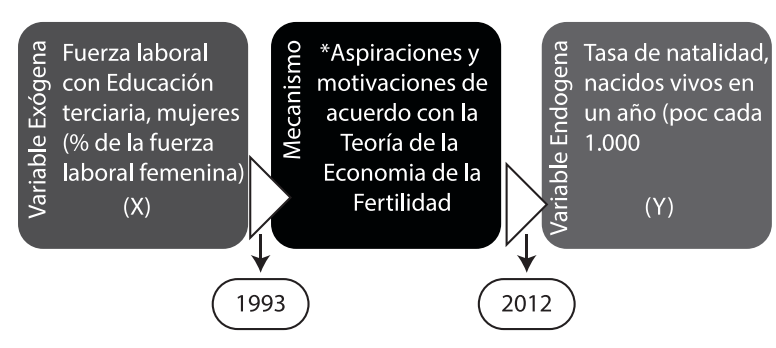

Figura 1. Representación del proceso de Análisis de los supuestos

\section{FALSACIÓN}

Una vez obtenidos los datos (19) (Ver Apéndice) y establecido el modelo $ß$ derivado lógicamente de la Teoría Económica de la Fertilidad, se procedió en primera instancia a corroborar el supuesto de normalidad, analizando los datos de las variables con los coeficientes de Asimetría típica y Curtosis, los cuales son mostrados en la Figura 2.

Dichos resultados se encuentran dentro del rango recomendado para asumir normalidad $(-2,2)$. Una vez comprobados los supuestos se procede a falsar a través del análisis de regresión simple. Los datos fueron analizados en el programa estadístico SPSS con el objetivo de verificar la asociación derivada de las variaciones en las variables: Fuerza laboral femenina con educación terciaria (como \% de la fuerza laboral femenina) y Tasa de natalidad, nacidos vivos en un año (por cada 1.000 personas), dentro del espacio de la Unión Europea (elegido por poseer los datos de la variable fuerza laboral femenina con educación terciaria, desde los años $80^{\prime}$, lo que no se cuenta para los países de América Latina).

En la Tabla 3 se muestran los resultados del modelo de regresión simple aplicado:

\section{TABLA 3. RESUMEN DEL MODELO}

\begin{tabular}{|rr}
\hline Modelo & 1 \\
\hline $\mathbf{R}$ &, 824 \\
\hline $\mathbf{R}$ cuadron &, 679 \\
\hline R cuadrado ajustado &, 669 \\
\hline Error estándar de la estimación &, 6663644382 \\
\hline
\end{tabular}

a. Predictores: (Constante), FUERZALAB

Nota: Resultados que se obtuvieron una vez realizadas las operaciones en el programa estadístico SPSS sobre las variables fuerza laboral femenina con educación terciaria (como \% de la fuerza labora femenina) y Tasa de natalidad, nacidos vivos en un año (por cada 1.000 personas), dentro del espacio de la Unión Europea, durante los años 1999 al 2012 (con la data obtenida del Banco Mundial).

En la Tabla 4 se muestran los resultados de la correlación de las variables de estudio (de acuerdo a resultados del programa estadístico):

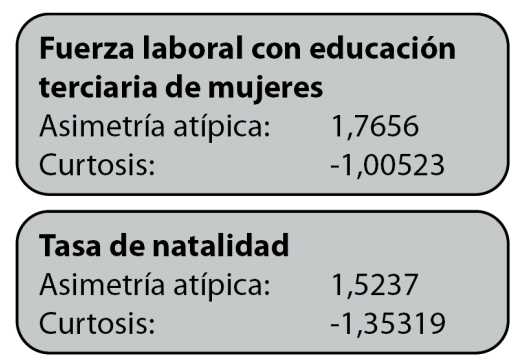

Figura 2: Supuesto de Normalidad de los datos obtenidos del Banco Mundial de las variables en estudio, durante los años 1999 al 2012 en la Unión Europea

TABLA 4. COEFICIENTES DE CORRELACIÓN DE LAS VARIABLES DE ESTUDIO

\begin{tabular}{|c|c|c|c|c|}
\hline \multirow[t]{2}{*}{ MODELO } & & $\begin{array}{l}\text { EFICIENTES NO } \\
\text { TANDARIZADOS }\end{array}$ & $\begin{array}{r}\text { COEFICIENTES } \\
\text { ESTANDARIZADOS }\end{array}$ & \multirow[t]{2}{*}{ T SIG } \\
\hline & B & Error Standar & Beta & \\
\hline 1 (Constante) & 14,67 & 0,41 & & 35,80, 000 \\
\hline FUERZALAB & $-0,16$ & 0,02 & $-0,82$ & -8,10, 000 \\
\hline
\end{tabular}

a. Variable dependiente: TASANATALIDAD

Nota: Resultados que se obtuvieron una vez realizadas las operaciones en el programa estadístico SPSS sobre las variables Fuerza laboral femenina con educación terciaria (como \% de la fuerza laboral femenina) y Tasa de natalidad, nacidos vivos en un año (por cada 1.000 personas), dentro del espacio de la Unión Europea, durante los años 1999 al 2012 (con la data obtenida del Banco Mundial).

El coeficiente de correlación simple (0.824) permite establecer que existe relación estadística entre las variables analizadas. Una relación moderadamente fuerte. Con relación al coeficiente $\beta$, se puede observar que tiene signo negativo, lo que supone una relación inversa entre las variables, mientras la fuerza laboral con educación terciaria femenina crece, la tasa de natalidad baja. Se puede constatar que $\mathrm{p}$-value es mucho menor a 0.05 , por lo tanto se concluye que es diferente de cero, con un $95 \%$ de nivel de confianza.

\section{CONCLUSIONES}

La Hipótesis Nula H0: $\mathrm{b}=0$ se rechaza, debido a que el p-value es menor que el nivel de significancia propuesto que es 0,05 .

Por lo tanto se acepta la hipótesis alternativa que $b \neq 0$, admitiéndose el supuesto propuesto en $\beta$.

Finalmente se corrobora que existe causalidad inversa en el modelo propuesto entre la variable exógena Fuerza laboral con educación terciaria, mujeres (\% de la fuerza laboral femenina) y la variable endógena Tasa de natalidad, nacidos vivos en un año (por cada 1.000 personas).

Los resultados de esta investigación son congruentes con investigaciones anteriores 
que han abordado el tema en otros contextos (Picchio, 1994), (Marín, 2002), (Alvis-Guzman et al., 2010), (Bonilla et al., 2009), (Mesa y Junca, 2011). Los diferentes estudios consultados como parte de la revisón de la literatura, han investigado el tema en otras realidades y con otras variables adicionales. Este estudio se ha enmarcado en una revisión histórica con data del Banco Mundial, en el contexto de la Unión Europea, en un período de 19 años (1999-2012). Tuvo como objetivo probar la correlación de las variables endógena y exógena, utilizando la metodología Alpha Beta (Figueroa, 2012), que se fundamenta en la teoría popperiana.

\section{REFERENCIAS BIBLIOGRÁFICAS}

Aguinaga, J. (1983). Hipótesis causales sobre fecundidad: el papel preponderante de la unidad familiar. REIS, 21(83), 83-101.

Álvarez, G. (2002). Decisiones de fecundidad y participación laboral de la mujer en España. Investigaciones Económicas, 26(1), 187-218.

Alvis-Guzmán, N., Yánez-Contreras, M., QuejadaPérez, R., Acevedo-González, K., y Del Río-Carrasquilla, F. (2010). Fecundidad y participación de la mujer en el mercado laboral en la Costa Caribe y en Colombia. Revista Cerencia y Políticas de Salud, 9(19), 90-107.

Anzorena, C. (2009). El ¿retorno? del "Tratado sobre la Familia” de Gary Becker. Algunas reflexiones en torno a los criterios de eficiencia que legitiman los planes compensatorios de fines de la década de 1990. KAIROS Revista de Temas Sociales, 13(24), 1-15.

Becker, G. (1960). An Economic Analysis of Fertility. New York: Publicaciones de la Universidad de $\mathrm{Co}^{-}$ lumbia y National Bureau of Economic Research. Recuperado de http://www.nber.org/chapters/ c2387.pdf

Becker, G. (1965). A Theory of the Allocation of time. The Economic Journal, 75(299), 493-517.

Becker, G. (1981). A treatise on the family. Estados Unidos: Publicaciones dela Universidad de Harvard.

Becker, G., \& Barro, R. (1986). A reformulation of the economic theory of fertility. Cambridge: National Bureau of Economic Research (Working Paper No. 1793).

Bonilla, G., García, E., y Mendoza, O. (2009). Impacto de las condiciones socioeconómicas familiares en las decisiones de fertilidad (número de hijos), utilizando un modelo de regresión Poisson: período de estudio 2005-2006. Paper de la ESPOL. Recuperado de http://www.dspace.espol.edu.ec/ bitstream/123456789/4896/1/7684.pdf
Para poder responder la interrongante planteada, sobre si la mujer activa tiene absoluta decisión en la fecundidad, se tendría que ampliar el estudio. En ese caso deben considerarse variables adicionales, como por ejemplo las relacionadas al matrimonio, a la actividad laboral del esposo, a las características familiares anteriores al matrimonio (costumbres, tradiciones sociales), el tipo de actividad empresarial desarrollada por la mujer, entre otras. Por ello, este estudio se puede considerar como base para la realización de otras investigaciones que consideren agregar más variables dependientes para relacionar la decisión sobre la fecundidad.

Bratti, M. (2003). Labor Force Participation and Marital Fertility of Italian Women: The Role of Education. Journal of Population Economics, 16(3), 525-554.

Cáceres, A., Elías, S., y Fernández, R. (2005). Fertilidad, Capital Humano y Crecimiento Económico: un análisis empírico. Progresos en Economía, Publicaciones de la Asociación Argentina de Economía Política. Recuperado de http://www. aaep.org.ar/anales/works/works2005/caceres_elias_fernandez.pdf

Figueroa, A. (2012). The Alpha-Beta Method. Scientific Rules for Economics and the Social Sciences. Buenos Aires: Cengage Learning.

Marín, M. R. (2002). Tasa de actividad femenina y fecundidad. ¿Ser madre o ser activa? Boletín ICE económico, 2723, 21-28.

Mesa, C. A., y Junca, G. A. (2011). Análisis de reducción de la fecundidad en Colombia: Modelo de determinantes próximos. Cuadernos de Economía, 30(54), 127-150.

Picchio, A. (1994). El trabajo de reproducción; tema central en el análisis del mercado laboral. En C. Borderías, C. Carrasco, \& A. Carmen (Eds.), Las mujeres y el trabajo: algunas rupturas conceptuales (pp. 451-490). Barcelona, España: Icaria.

Rindfuss R., Morgan, P., \& Offutt, K. (1996). Education and the Changing Age Pattern of American Fertility: 1963-1989. Demography, 33(3), 277-290.

Tijerina, E. (1999). Aprendiendo Economía con los Nobel. 1969-1998. México, D.F.: Plaza y Valdés Editores. 